\title{
Visual estimation of apertures for wheelchair locomotion in novices: perceptual judgment and motor practice
}

\author{
Sérgio T. Rodrigues, Nicole C. Galvão, and Gisele C. Gotardi \\ Universidade Estadual Paulista, Bauru, SP, Brazil
}

\begin{abstract}
The aim of the present study was to determine the effects of motor practice on visual judgments of apertures for wheelchair locomotion and the visual control of wheelchair locomotion in wheelchair users who had no prior experience. Sixteen young adults, divided into motor practice and control groups, visually judged varying apertures as passable or impassable under walking, pre-practice, and post-practice conditions. The motor practice group underwent additional motor practice in 10 blocks of five trials each, moving the wheelchair through different apertures. The relative perceptual boundary was determined based on judgment data and kinematic variables that were calculated from videos of the motor practice trials. The participants overestimated the space needed under the walking condition and underestimated it under the wheelchair conditions, independent of group. The accuracy of judgments improved from the pre-practice to post-practice condition in both groups. During motor practice, the participants adaptively modulated wheelchair locomotion, adjusting it to the apertures available. The present findings from a priori visual judgments of space and the continuous judgments that are necessary for wheelchair approach and passage through apertures appear to support the dissociation between processes of perception and action. Keywords: visual judgment, aperture estimation, time-to-passage, wheelchair, locomotion.
\end{abstract}

Received 02 March 2014; received in revised form 29 June 2014; accepted 03 July 2014. Available online 25 November 2014.

\section{Introduction}

Humans and other animals are continuously judging space based on visual information that is available in the environment. We often have to avoid potential obstacles and collisions by altering our whole-body trajectory and adjusting the position of the head and body segments to overcome apertures or gaps between objects. Although we easily manage to successfully perform our everyday activities, how our perception and action systems interact during learning and adaptation has been a topic of debate (Kunz, Creem-Regehr, \& Thompson, 2013). In particular, wheelchair locomotion has an additional perceptual-motor requirement because the wheelchair represents an extension of the user's body that needs to be perceived and controlled (Stoffregen, Yang, Giveans, Flanagan, \& Bardy, 2009).

People who can walk are able to properly estimate their spatial requirements. Warren and Whang (1987) found that young adults, when instructed to walk through apertures of various widths without touching the

Sérgio T. Rodrigues, Nicole C. Galvão, and Gisele C. Gotardi, Laboratory of Information, Vision, and Action (LIVIA), Department of Physical Education, Universidade Estadual Paulista (UNESP). Correspondence regarding this article should be directed to: Sérgio Tosi Rodrigues - Universidade Estadual Paulista (UNESP), Av. Luis Edmundo Carrijo Coube, 14-01, Vargem Limpa, Bauru, SP, 17033-360, Brazil. Email: srodrigu@fc.unesp.br lateral frames, rotated their trunk if the aperture was less than 1.3-times the width of their shoulders. However, when asked to judge from a distant position whether they could pass through the aperture, they indicated a width that was 1.16-times larger than the width of their shoulders. This suggests that the participants could correctly perceive the size of apertures with respect to their own bodies, and this relationship (termed the relative perceptual boundary $[\mathrm{RPB}]$ ) was greater than 1.0, showing that individuals tend to slightly overestimate spatial requirements to provide a safety margin (Higuchi, Takada, Matsuura, \& Imanaka, 2004).

Relatively skilled wheelchair users have made judgments of spatial requirements that are comparable to walkers. Nonhandicapped adults who are well trained in wheelchair locomotion presented an RPB of 1.06 (Flascher, Shaw, Kader, \& Aromin, 1995). Children with Cerebral palsy who normally used wheelchairs, with a minimum experience of 2 years, were instructed to make judgments of the passable minimal aperture width. The older group (9-13 years of age) judged it to be approximately 1.1-times the wheelchair width, whereas the younger group (5-8 years of age) judged it to be approximately 1.3 (Savelsbergh, Dekker, Vermeer, \& Hopkins, 1998). This improved accuracy in older children may suggest an influence of extended experience. In addition to the role of experience on the accuracy of judgments, a relevant methodological issue is the definition of the RPB to allow comparisons 
across studies. Higuchi et al. (2004) noted that distinct notions of "maximum aperture width as impassable" and "minimum aperture width as passable" could result in a difference of approximately $5 \mathrm{~cm}$ among studies. These authors suggested applying a typical psychophysical method based on the proportion of passable judgments as a function of aperture widths and defining the perceptual boundary as the value of the aperture width at which the function passes through the $50 \%$ level. Based on this definition, they recalculated the RPB from previous studies. Walkers presented an RPB of 1.21 from the second experiment of Warren and Whang (1987). Skilled wheelchair users showed an RPB of 1.02 from Flascher et al. (1995) and 1.03 from Savelsbergh et al. (1998). This confirms that walkers and skilled wheelchair users similarly judge and reduce risks, although skilled wheelchair users adopt a smaller safety margin (Higuchi et al., 2004).

Novice wheelchair users, judging the narrowest aperture through which they could pass, also presented a consistent ratio between the possible apertures and the wheelchair width, approximately 1.20 (Shaw, Flascher, \& Kadar, 1995). Higuchi et al. (2004) used a task of judging the minimum width of a passable aperture in novices. One group of participants was given 1014 trials of motor practice (experiment 1) using the wheelchair to pass through apertures of varying widths. The participants overestimated their ability to pass through apertures, showing a mean ratio of aperture width to wheelchair width of .93 across groups. The accuracy of judgments did not improve after motor practice of passing through apertures. Stoffregen et al. (2009) studied novice wheelchair users who had to judge the vertical aperture through which they could pass and found distinct results regarding motor experience with the wheelchair. Prior to making the judgments, some of the participants (locomotor practice group) were given brief (2-3 min) practice with self-controlled wheelchair locomotion. The other participants (specific practice group) had additional specific practice, going back and forth under an apparatus that limited the vertical aperture four times with different heights. Interestingly, these participants who practiced made more accurate judgments, even when the practice was general and distinct from the judgment situation. The participants who had no practice gave inaccurate judgments. Additionally, this brief practice strongly influenced exploratory movements of the head and trunk during judgment sessions, which were interpreted as facilitating accurate judgments (Stoffregen et al., 2009).

In short, the reviewed literature has shown that experience in walking and using wheelchairs appears to be related to more accurate judgments of apertures. In contrast, previous studies with novices indicated that passing through apertures several times during relatively brief motor practice did not improve the participants' knowledge of their ability to pass through apertures. However, controversy exists with regard to how motor practice and perceptual judgments interact, as shown by the results from Stoffregen et al. (2009). In the present study, the accuracy of aperture judgments pre- and post-motor practice in participants with no previous experience was compared. Additionally, a walking condition was included as a control for judgment experience based on the participants' own body and based on the wheelchair width.

One aspect that may be a factor in this debate is the nature of the motor practice and visuomotor requirements that are involved in this practice. The regulation of space and time while approaching and crossing an aperture throughout the practice period requires continuous visual judgments. This offers an opportunity for wheelchair users to better know the environmental spatial characteristics, wheelchair features and possibilities, and motor control parameters that are necessary for this type of locomotion. Perception-action analysis based on time-to-passage visual information that is available to wheelchair users is a novel feature that was included in the present study in an attempt to provide detailed information about motor practice and its possible changes associated with the visual judgments of apertures. During motor practice, the kinematic features of the wheelchair approach phase and its final deceleration prior to crossing an aperture are based on continuous judgments of the aperture to control locomotion and may interact with the capabilities associated with a priori judgments further away from the aperture.

The locomotor optic flow field (Gibson, 1966, 1979) that results from the relative approach to an obstacle contains information about the time that remains until the obstacle reaches the eye's plane. This visual information has been called time-to-collision, timeto-contact, or time-to-passage (e.g., Lee, 1998; Lee, Young, \& Rewt, 1992; Wann, Edgard, \& Blair, 1993). The rate of dilation of the optical subtended angle of the object, named tau (Lee, 1976), is inversely proportional to the time remaining until the object is reached if the approaching velocity is constant. More recently, this variable has been generalized to the notion of closure of a gap between the observer and the obstacle (Lee, 1998, 2009). Sensitivity to the variables tau and tau-dot (e.g., Kim, Turvey \& Carello, 1993; Sun \& Frost, 1998) and their use during the control of actions have been confirmed in various situations, such as the braking of a car (Lee, 1976; Treffner, Barrett, \& Petersen, 2002; Treffner, Barrett, Petersen, \& White, 2002), a bird docking to feed (Lee, Young, Reddish, \& Rand, 1991), and a somersaulter landing (Lee et al., 1992). The limitations of this strategy and alternative sources of information have been debated by researchers in the field (e.g., Tresilian, 1994, 1999; Wann, 1996). With regard to the control of deceleration and braking actions, Lee (1976) demonstrated that the tau rate of change in time (i.e., its first derivative in time, termed tau-dot) can inform on the possibility of collisions. More specifically, maintaining tau-dot around the critical value of -.5 is necessary to distinguish situations in which the current deceleration is sufficient to avoid a collision that will occur (tau-dot $\geq-.5$ ) from situations in which it will not 
occur (tau-dot < -.5). In summary, Lee (1976) proposed that humans and other animals use tau and tau-dot to start the deceleration/braking action and regulate its intensity, respectively. These concepts were adopted in the present study, and wheelchair kinematics were recorded to analyze the visual control of the wheelchair to cross distinct apertures throughout the practice phase.

Previous studies have shown that the magnitudes of tau and tau-dot were unaffected by different initial velocities or types of trajectories (Rodrigues, Bertoloni, Denardi, \& Ferracioli, 2006; Rodrigues, Schiavon, \& Macegoza, 2012) during the final deceleration to stop a bicycle by recreational cyclists. Regarding expertise, elite long jumpers regulated their final three steps' spatial variability prior to jumping based on time-tocontact information (Lee, Lishman, \& Thompson, 1982). Similarly, non-jumpers, novices, and expert long jumpers did not essentially differ in their timeto-contact-based visual strategy to jump (Scott, Li, \& Davids, 1997). Altogether, the time-to-passage studies reviewed above suggest that tau and tau-dot are relative variables that remain constant across changes in contexts, control parameters, and experience levels.

The aim of the present study was to determine the effects of motor practice on visual judgments of apertures for wheelchair locomotion and the visual control of wheelchair locomotion in wheelchair users with no prior experience. Our hypothesis was that motor practice would improve the overall accuracy of aperture judgments between the pre-practice and post-practice conditions only in the practice group. Additionally, judgments in the walking condition were expected to be more accurate than in the wheelchair conditions. The use of time-to-passage analysis was exploratory in the sense that no prior studies have evaluated the effects of motor practice on the visuomotor control of wheelchairs and visual judgments of apertures. Tau and tau-dot were expected to not differ throughout the practice blocks or across distinct apertures and kinematic variables were expected to reveal adaptability to the space that was available in each aperture. The variables that were related to the overall outcome of wheelchair locomotion were also expected to reflect improvements in performance.

\section{Methods}

\section{Participants}

Sixteen undergraduate students (mean age $=22.17$ years, SD $=1.98$ years) with no experience in using wheelchairs volunteered to participate in this study. The participants were equally and randomly divided into motor practice and control groups. Informed consent was obtained from each participant prior to the experiment, and the local University Ethics Committee approved the procedures used in this study.

\section{Apparatus}

Stationary visual judgments were made while the participants sat on a sportive wheelchair $(90 \mathrm{~cm}$ width,

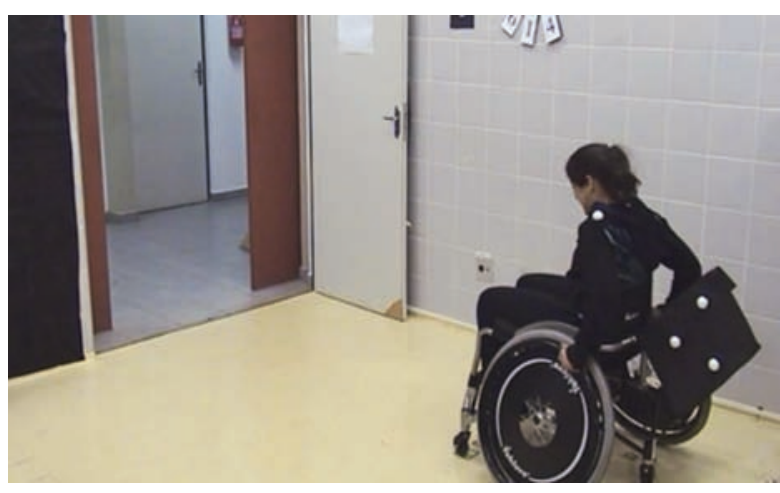

Figure 1. Video frame showing a participant in the practice group during wheelchair locomotion toward the aperture. The participant was wearing black clothes to facilitate digital recognition of the markers (one on the participant's left acromion and three on the back of the wheelchair).

$45 \mathrm{~cm}$ height at seat pan) or regular chair (45 cm height at seat pan). An apparatus made of two moveable wood panels was regulated to present different horizontal apertures during the experimental sessions. Figure 1 shows a participant during wheelchair locomotion who was recorded with a Sony DCR DVD 405 video camera $(60 \mathrm{~Hz})$. The images of each trial were clipped, bidimensionally reconstructed, and exported in space coordinates of the tracked markers (Software APAS, Ariel Dynamics, version 1). One marker was placed on the participant's left shoulder (acromion) to represent the participant's eye plane, and three markers were placed on the back of the wheelchair to represent its motion plane. Matlab (Mathworks 2006, version 7.10) and SPSS (1999, version 9) software was used to calculate the dependent variables and perform the statistical analyses, respectively.

\section{Visual estimation task}

The participants were $2.8 \mathrm{~m}$ away from the aperture and had to judge whether the aperture was passable without touching it or impassable. Judgments were made with respect to two distinct imagined situations: "walking” and "wheelchair." In the walking situation, the participants sat on a regular chair and judged whether apertures that ranged from $25 \mathrm{~cm}$ to $65 \mathrm{~cm}$ (at 5 -cm intervals) were passable without rotating their shoulders. In the wheelchair situation, they sat in the wheelchair and judged whether apertures that ranged from $65 \mathrm{~cm}$ to $120 \mathrm{~cm}$ (at 5-cm intervals) were passable with possible changes in arm posture to reduce their spatial requirements while necessarily grasping the hand-rims. The participants were free to move their head, body, and arms but remained seated.

\section{Procedures}

The protocol was divided into five parts as suggested by Higuchi et al. (2004). The practice group was subjected to (i) visual estimation of different apertures in the "walking" situation, (ii) familiarization 
with the wheelchair for $5 \mathrm{~min}$, (iii) pre-practice visual estimation of different apertures in the "wheelchair" situation, (iv) motor practice with the wheelchair, passing through different apertures, and $(v)$ postpractice visual estimation of different apertures in the "wheelchair" situation. The control group underwent the same protocol without item iv above. They had the same time interval between items iii and $v$. The order was identical for both groups. During rest or no-activity intervals, the participants were blindfolded to avoid estimations.

During the three conditions of estimation (walking, wheelchair pre-practice, and wheelchair post-practice), different apertures were presented to the participants according to the method of limits, in which a series of aperture widths was presented in either an ascending or descending order with consecutive intervals. The series terminated when a participant gave two successive "passable" responses in the ascending order or two successive "impassable" responses in the descending order. Each participant began with the ascending order of apertures and kept alternating between descending and ascending orders until completing three ascending series and three descending series in each condition.

During the five-minute familiarization period, the participants tried moving with the sportive wheelchair and were instructed to pay attention to their arm position and wheel inclination. They were not allowed to pass through the aperture. During the motor practice period, the participants passed through the apertures, performing 10 blocks with five trials each with five different apertures $(85,90,95,100$, and $105 \mathrm{~cm})$ that were randomly presented. In each trial, the participants started to move the wheelchair from an initial distance of $2.8 \mathrm{~m}$ and tried to pass through the aperture without touching it. In cases of judging the aperture to be too small to pass, they were instructed to stop and move backward. These trials were considered missed. The participants consistently stopped during the trials with an $85 \mathrm{~cm}$ aperture, and so this level was excluded from the analysis. Missing trials for all of the other apertures were replaced by the mean of that aperture. Practice blocks 1, 5, and 10 (five trials each) were recorded for further kinematic analysis.

\section{Dependent variables}

Perceptual judgments. The perceptual boundary (PB) was determined by the estimations in the three conditions (walking, wheelchair pre-practice, and wheelchair post-practice) to make comparison between groups. To obtain the PB for each participant in each condition, the proportions of passable judgments (vertical axis) were plotted as a function of aperture widths (horizontal axis). The psychometric functions for each participant in each condition were adjusted using the Psignifit, version 2.5.6, toolbox for Matlab (http:// bootstrap-software.org/psignifit; accessed June 26, 2014), which implements the "maximum-likelihood" method described by Wichmann and Hill (2001). The PB was defined as the value on the horizontal axis at which the curve crossed the level of .5 (equivalent to $50 \%$ ). To normalize the data between participants and conditions, PB values were divided by the width between the shoulders in the walking condition and by the wheelchair width $(90 \mathrm{~cm})$ in the wheelchair pre- and post-practice conditions, generating the RPB variable (Figure 2).

Kinematic variables measured during the practice phase. Wheelchair locomotion performance (error rate [ER]) was defined as the number of times a participant in the motor practice group performed a motor error (i.e., touching the wood panel that defined the aperture). To determine when a participant touched the panel, the experimenter analyzed the video recording to confirm the visual observation and asked the participant to report the mistake.

For the kinematic analysis, each trial that was recorded in a digital video format was transformed to AVI format to run bidimensional reconstruction of the data. The wheelchair movement data with respect to the aperture were filtered with a low-pass, fourth-order Butterworth filter, with a frequency cut-off set to $1 \mathrm{~Hz}$, according to the residual analysis suggested by Winter (1990).

Movement time (MT) was defined in the motor practice trials as the interval between the onset of movement toward the aperture passage and moment at which the wheelchair completely passed through the aperture. The mean velocity (MV) of wheelchair movement was the ratio between the distance travelled by the wheelchair and MT. The peak velocity (PV) was the highest magnitude of velocity during forward wheelchair movement.

Based on the wheelchair kinematic data, additional variables (Figure 3 ) were determined to analyze the visual control involved in deceleration with respect to the aperture during motor practice trials: velocity at deceleration onset (VON), distance from the wheelchair to the aperture at deceleration onset (DIST), tau margin at deceleration onset (TAU), slope of a linear regression of the tau margin as a function of time during the deceleration phase (termed tau-dot [DOT]), and duration of the deceleration phase (DUR).

\section{Statistical analysis}

Visual estimation data (PB and RPB) were subjected to a group (motor practice, control) $\times$ condition (walking, wheelchair pre-practice, wheelchair post-practice) analysis of variance (ANOVA), with repeated measures in the last factor. Wheelchair locomotion performance (ER) was subjected to a one-way ANOVA, with practice block (1 to 10) as the factor. The variables based on the kinematic data (MT, MV, PV, VON, DIST, TAU, DOT, and DUR) were subjected to a practice block $(1,5,10) \times$ aperture (90, 95, 100, 105) ANOVA, with repeated measures in both factors. Tukey Honestly Significant Difference tests, 
Partipant: p4

Group: Motor Practice

RPB: Relative Perceptual Boundary

Condition: WHEELCHAIR (PRE-PRACTICE)

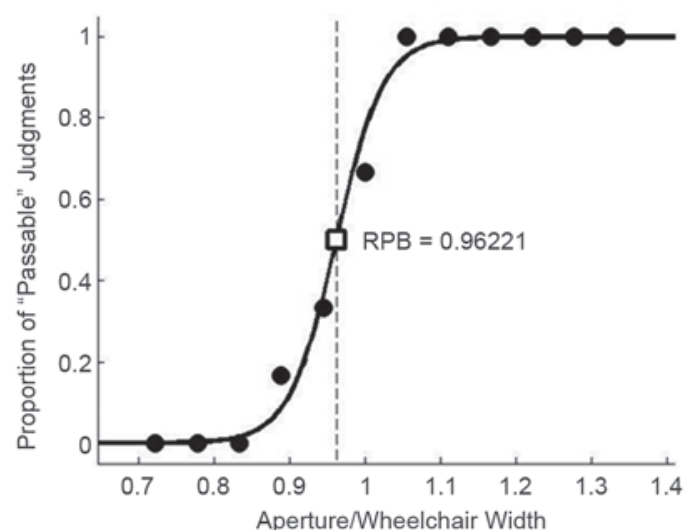

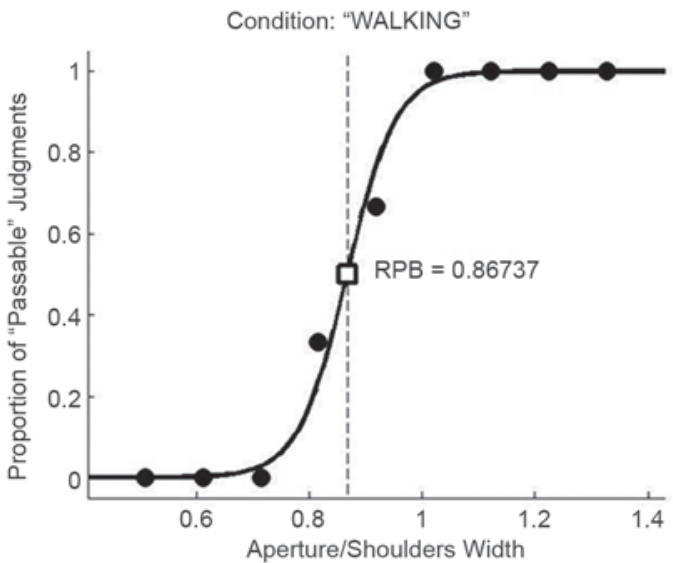

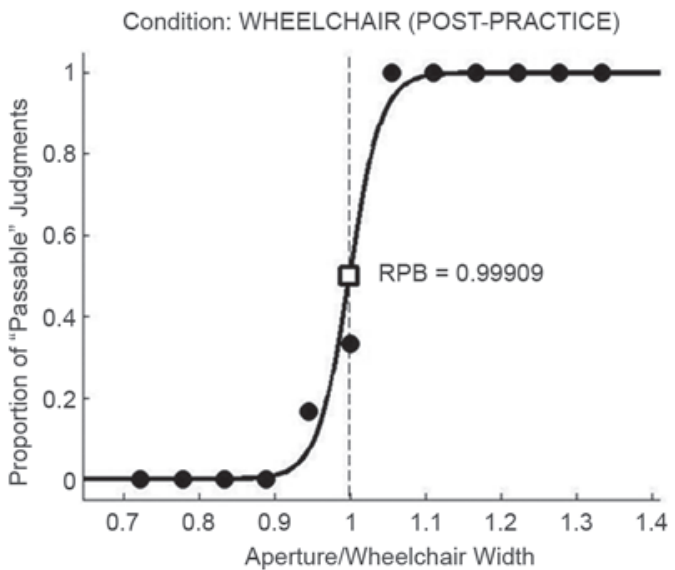

Figure 2. Illustration of the determination of the relative perceptual boundary for walking (upper right), wheelchair pre-practice (bottom left), and wheelchair post-practice (bottom right) conditions in a practice group participant.

Greenhouse-Geisser degrees of freedom adjustments, and Bonferroni multiple-comparison probability adjustments were conducted as necessary (Maxwell \& Delaney, 1990). The significance level was .05 for all of the analyses.

\section{Results}

\section{Visual estimation of apertures}

The PB was significantly affected by condition $\left(F_{2,28}\right.$ $=271.00, p<.001)$. The post hoc tests revealed that the $\mathrm{PB}$ was greater in the wheelchair pre- and post-practice conditions than in the walking condition (both $p<.001$ ), and the $\mathrm{PB}$ was greater in the post-practice condition than in the pre-practice condition $(p=.006)$. Similarly, the RPB was significantly affected by condition $\left(F_{2,28}=\right.$ 25.03, $p<.001$ ), with greater values in the wheelchair conditions than in the walking condition (both $p<.001$ ), and greater values were observed in the post-practice condition than in the pre-practice condition $(p=.006$; Table 1).

\section{Visual control during motor practice}

During motor practice, the number of errors (ER) was significantly reduced throughout the blocks $\left(F_{9,63}=\right.$ 2.74, $p=.048)$. Block $1(M=.88, S E=.23)$ had the highest ER compared with all of the other blocks, which had mean values < .38 (Figure 4). Table 2 summarizes all of the other variables obtained from the motor practice trials. MT was significantly affected by aperture $\left(F_{3,21}=\right.$ 27.66, $p<.001)$. The post hoc tests revealed significant differences between apertures of 90 and $95 \mathrm{~cm}(p=$ $.042), 90$ and $100 \mathrm{~cm}(p=.008), 90$ and $105 \mathrm{~cm}(p=$ $.020)$, and 95 and $105 \mathrm{~cm}(p=.002)$. Similarly, MV and PV were significantly affected by aperture $\left(F_{3,21}=31.81\right.$ and 25.52, respectively, both $p \leq .001)$. The post hoc tests for MV revealed significant differences between apertures of 90 and $95 \mathrm{~cm}(p=.017), 90$ and $100 \mathrm{~cm}(p$ $=.005), 90$ and $105 \mathrm{~cm}(p=.010)$, and 95 and $105 \mathrm{~cm}$ $(p=.003)$. For PV, significant differences were found between apertures of 90 and $100 \mathrm{~cm}(p=.006), 90$ and $105 \mathrm{~cm}(p=.004), 95$ and $105 \mathrm{~cm}(p<.001)$, and 95 and $105 \mathrm{~cm}(p=.018)$.

With regard to the control of final deceleration (Table 2), DIST and VON were significantly affected by aperture $\left(F_{3,21}=9.18\right.$ and 14.42 , respectively, both $p \leq$ .002). The post hoc tests for DIST revealed significant differences between apertures of 90 and $105 \mathrm{~cm}$ ( $p=$ $.042), 95$ and $100 \mathrm{~cm}(p=.041)$, and 95 and $105 \mathrm{~cm}(p$ $=.005)$. For VON, significant differences were found between apertures of 90 and $100 \mathrm{~cm}(p=.013), 90$ and 
Participant/Block/Trial: p622

MT: $5.3667 \mathrm{~s}$

MV: $0.5426 \mathrm{~m} / \mathrm{s}$

PV: $0.815 \mathrm{~m} / \mathrm{s}$

VON: $0.815 \mathrm{~m} / \mathrm{s}$

DIST: $0.34507 \mathrm{~m}$

TAU: $0.4234 \mathrm{~s}$

DOT: -0.57342

DUR: $0.68333 \mathrm{~s}$
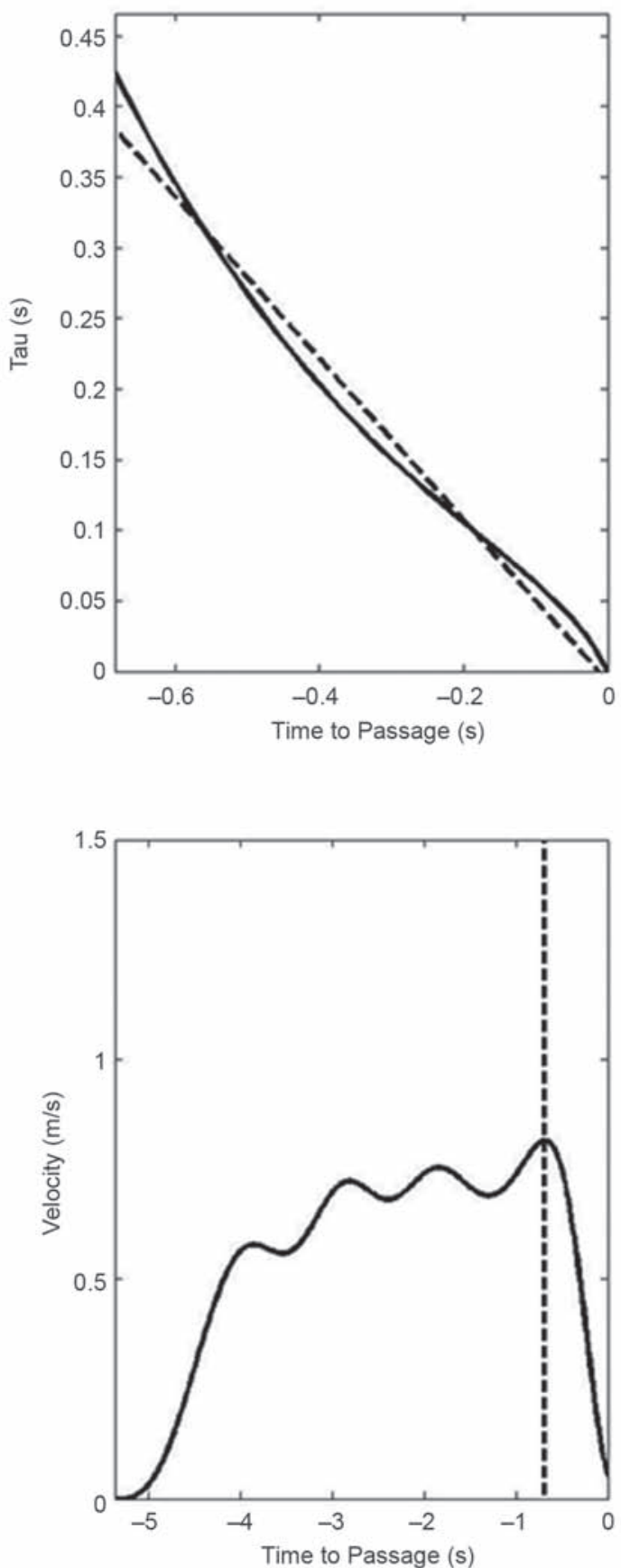

Figure 3. Illustration of the determination of dependent variables based of kinematic data. It shows the participant's identification and values of each variable in that trial (top left), wheelchair position (bottom left), and velocity (bottom right) as a function of time to passage during all of the trials (vertical dashed lines represent the onset of deceleration) and tau (solid line) as a function of time to passage (top right) during the deceleration phase (the dashed line resulted from the linear regression of tau's data; its slope equals tau-dot).

Table 1. Mean (standard error) of perceptual boundary (PB) and relative perceptual boundary (RPB) in the walking, wheelchair pre-practice, and wheelchair post-practice conditions in the motor practice and control groups.

\begin{tabular}{llll}
\hline Group & Condition & PB $(\mathbf{c m})^{\mathrm{a}, \mathrm{b}}$ & $\mathbf{R P B}^{\mathrm{a}, \mathrm{b}}$ \\
\hline \multirow{3}{*}{ Control } & Walking & $46.31(2.69)$ & $1.04(.04)$ \\
& Wheelchair Pre-Practice & $78.87(2.07)$ & $.87(.02)$ \\
& Wheelchair Post-Practice & $80.23(2.50)$ & $.89(.02)$ \\
\multirow{2}{*}{ Motor Practice } & Walking & $47.67(2.69)$ & $1.08(.04)$ \\
& Wheelchair Pre-Practice & $81.89(2.07)$ & $.91(.02)$ \\
& Wheelchair Post-Practice & $85.80(2.50)$ & $.95(.02)$ \\
\hline
\end{tabular}

${ }^{a}$ Significant effect of condition $(p<.001)$. ${ }^{\mathrm{b}}$ All pairwise comparisons between conditions were significant $(p<.01)$. 


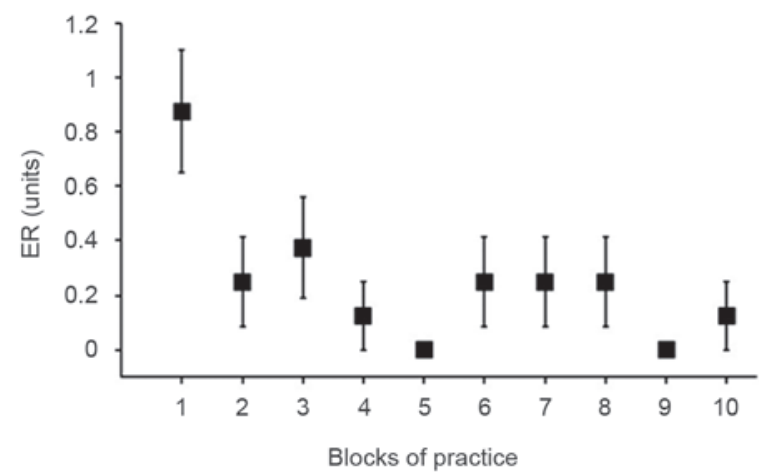

Figure 4. Motor errors in wheelchair locomotion (error rate [ER]) as a function of blocks of practice.

$105 \mathrm{~cm}(p=.007)$, and 95 and $105 \mathrm{~cm}(p<.025)$. The main effect of block on DUR was significant $\left(F_{2,14}=\right.$ $7.80, p=.013)$. No significant pairwise comparisons were observed between blocks. The optical variables TAU and DOT were also significantly affected by block $\left(F_{2,14}=6.62\right.$ and 5.21, respectively, both $p \leq$ .036). No pairwise comparisons between blocks were significant. All of the other main effects and interactions are not reported because they did not reach the level of significance.

\section{Discussion}

The purpose of the present study was to analyze the effects of motor practice on visual judgments of apertures for wheelchair locomotion and the visual control of wheelchair locomotion in wheelchair users with no prior experience. With regard to the judgment of apertures when a tool is used as an extension of the body (e.g., a wheelchair), we hypothesized that judgments in the walking condition would be more accurate than in the wheelchair conditions. The accuracy of judgments can be observed from the magnitude of the RPB, which equals the absolute PB divided by the shoulder width in the walking condition or wheelchair width in the wheelchair pre- and post-practice conditions. A magnitude of 1.0 indicates that the participants precisely judged the space available in a given aperture width as passable, coinciding with the perceptual decision for a physical structure in the environment (Figure 2). Differences in judgments between the walking condition and wheelchair conditions were assumed to indicate an effect of the experience and familiarity of judging with respect to the participants' own body size and the size of an external tool.

The results confirmed that judgments in the walking condition were more accurate than in the wheelchair conditions in both groups, as expected. Interestingly, the same significant effects of condition and pairwise comparisons between conditions were found for the RPB and PB variables (Table 1). RPB values in the walking condition were $>1.0$, indicating that the participants would not bump or touch the borders of the apertures, corroborating previous studies (Jiang \& Mark, 1994; Warren \& Whang, 1987). Walkers in the second experiment of Warren and Whang (1987) judged apertures to be 1.16-times larger (or 1.21-times larger after recalculation by Higuchi et al., 2004) than the width of their shoulders. The magnitudes of the RPB were $<1.0$, suggesting that motor practice did not modify judgments of the real available space. An RPB of approximately .9 revealed a perceptual mistake because the wheelchair is larger than the space that was judged as passable. These results are consistent with Higuchi et al. (2004) who found that participants overestimated the necessary space for walking and underestimated it in the wheelchair condition. These differences clearly indicate the difficulties that novice wheelchair users have in adapting their judgments to the spatial requirements created by the use of such a tool.

Table 2. Mean (standard error) of movement time (MT), mean velocity (MV), distance from the wheelchair to the aperture at deceleration onset (DIST), velocity at deceleration onset (VON), peak velocity (PV), duration of the deceleration phase (DUR), tau margin at deceleration onset (TAU), and tau-dot (DOT) for apertures of 90, 95, 100, and $105 \mathrm{~cm}$ during practice blocks 1, 5, and 10.

\begin{tabular}{|c|c|c|c|c|c|c|c|c|c|}
\hline Block & Aperture & $\operatorname{MT}(\mathbf{s})^{\mathrm{a}}$ & $\operatorname{MV}(\mathbf{m} / \mathbf{s})^{\mathrm{a}}$ & DIST (m) ${ }^{\mathrm{a}}$ & $\operatorname{VON}(\mathrm{m} / \mathrm{s})^{\mathrm{a}}$ & $\operatorname{PV}(\mathrm{m} / \mathrm{s})^{\mathrm{a}}$ & $\operatorname{DUR}\left(\mathbf{s}^{\mathrm{b}}\right.$ & TAU (s) ${ }^{\mathrm{b}}$ & DOT $^{\mathrm{b}}$ \\
\hline \multirow{4}{*}{1} & 90 & $8.63(.60)$ & $-.40(.20)$ & $.29(.06)$ & $.46(.03)$ & $.67(.04)$ & $.96(.15)$ & $.63(.12)$ & $-.602(.020)$ \\
\hline & 95 & $6.71(.33)$ & $-.49(.20)$ & $.22(.01)$ & $.59(.05)$ & $.76(.03)$ & $.74(.13)$ & $.41(.04)$ & $-.566(.020)$ \\
\hline & 100 & $5.52(.34)$ & -.61 (.30) & $.42(.08)$ & $.84(.06)$ & $.85(.04)$ & $.77(.14)$ & $.49(.10)$ & $-.583(.020)$ \\
\hline & 105 & $5.10(.22)$ & $-.63(.30)$ & $.34(.02)$ & .89 (.10) & $.97(.10)$ & $.68(.01)$ & $.42(.01)$ & $-.565(.005)$ \\
\hline \multirow{4}{*}{5} & 90 & 8.55 (.61) & $-.42(.20)$ & $.20(.01)$ & $.53(.02)$ & $.68(.03)$ & $.65(.01)$ & $.39(.01)$ & $-.547(.005)$ \\
\hline & 95 & $6.78(.50)$ & $-.50(.30)$ & $.24(.02)$ & $.63(.05)$ & $.82(.08)$ & $.59(.01)$ & $.37(.01)$ & $-.555(.002)$ \\
\hline & 100 & $5.44(.32)$ & $-.61(.30)$ & $.34(.01)$ & $.80(.04)$ & $.79(.04)$ & $.74(.05)$ & $.44(.02)$ & $-.552(.017)$ \\
\hline & 105 & $4.61(.18)$ & -.68 (.20) & $.41(.03)$ & $1.02(.10)$ & $.98(.08)$ & $.67(.01)$ & $.43(.01)$ & $-.565(.003)$ \\
\hline \multirow{4}{*}{10} & 90 & $7.17(.41)$ & $-.47(.20)$ & $.20(.01)$ & $.53(.03)$ & $.75(.08)$ & $.61(.02)$ & $.37(.01)$ & $-.555(.004)$ \\
\hline & 95 & $5.88(.18)$ & $-.56(.20)$ & $.29(.04)$ & $.65(.04)$ & $.77(.02)$ & $.70(.08)$ & $.47(.06)$ & $-.592(.021)$ \\
\hline & 100 & $5.16(.35)$ & $-.64(.40)$ & $.35(.04)$ & $.98(.16)$ & $.92(.06)$ & $.66(.01)$ & $.40(.01)$ & $-.564(.003)$ \\
\hline & 105 & $4.88(.24)$ & $-.66(.30)$ & $.40(.02)$ & $.97(.06)$ & $1.04(.09)$ & $.68(.01)$ & $.42(.01)$ & $-.568(.004)$ \\
\hline
\end{tabular}

${ }^{a}$ Significant effect of aperture $(p<.003)$; see text for details. ${ }^{b}$ Significant effect of block $(p<.05)$; no significant pairwise comparison between blocks. 
With regard to the relationship between motor experience and visual judgments, our hypothesis was that controlling the wheelchair during practice would improve the accuracy of judgments between the prepractice and post-practice conditions only in the motor practice group. The results showed that the accuracy of judgments improved from the pre-practice to postpractice condition in the motor practice group, but the same improvement in accuracy occurred in the control group. Two possible reasons may explain why motor practice did not improve the judgments of the aperture. The first explanation is related to the specificity of practice and learning. Both groups improved their judgments, showing an RPB closer to 1.0 in the second set of judgments, independent of motor practice. In the present study, the participants improved their judgments by repeating the judgments. This is consistent with previous studies in which the accuracy of judgments improved over the trials, despite the absence of motor practice (Mark, 1987; Mark, Balliet, Craver, Douglas, \& Fox, 1990; Stoffregen, Yang, \& Bardy, 2005).

The second explanation is based on the differences in the visual tasks involved in the judgment of the space or apertures (which involves only making judgments) and visual control of wheelchair locomotion (which involves performing the perceptual-motor action). These differences are related to distinct functions of the visual system that support two interrelated aspects in the use of a wheelchair: a priori judgment and continuous judgment. A priori judgments are necessary to establish a gross route that serves to create spatial goals of locomotion. Continuous judgments are made online and based on visual feedback (e.g., recent past information), and feedforward information about the near future serves to adjust wheelchair approach and passage through the aperture itself. Thus, when a participant is further away from the aperture, visual mapping (i.e., mental representation) is used to judge and plan the future direction to be followed. When judging the approach and passage through the aperture, continuous updating of the relationship between the user and the structural space that is available in the optic flow is necessary. In this context, tau and tau-dot may explain how vision (more specifically, the inverse of the image dilation rate on the retina) plays a role in the control of wheelchairs during the passage through apertures (Lee, 1998, 2009). Milner and Goodale (1995, 2008) proposed a two-visual-systems theory, which fits the notions of a priori and continuous judgments, respectively. The ventral (vision-for-perception) system is responsible for identifying aperture space. The dorsal (vision-for-action) system provides information in egocentric coordinates for movement control.

Regarding the analysis of the practice period with the wheelchair in the motor practice group, significant improvement in performance was indicated by the ER, with a higher number of mistakes only in the first block of practice (.88), with stabilization of performance from block 2 to block $10(<.38)$. All of the other variables based on the kinematic data of the wheelchair did not significantly affect the motor practice blocks (Table 2). In fact, the practice block factor had a main effect on DUR, TAU, and DOT, but none of the pairwise comparisons reached significance, which was interpreted as an inconsistent effect. The variables MT, MV, DIST, VON, and PV were systematically affected by the aperture width, revealing adaptability to the space that was available for each aperture. MT, $\mathrm{MV}$, and PV showed adaptation during all of the trials. When the aperture was larger, higher mean and peak velocities and a brief trial duration were observed. With small apertures, the opposite trend was observed. In the final deceleration phase, the variables DIST and VON showed adaptation to the apertures in which the position where deceleration onset occurred and velocity at this instant were modulated according to the aperture width. The perception of the availability of space for possible actions with the wheelchair in the present study can be expressed by the notion of "affordance." Affordances are potentials for action for a given animal in a given environment and influence the outcome of interactions with the environment, favoring adaptation (Gibson, 1979; Stoffregen et al., 2009).

The time-to-passage analysis (Figure 3) did not show changes in the visuomotor control of wheelchairs caused by motor practice. No variables were systematically affected by blocks of practice. However, this analysis revealed that tau and tau-dot did not differ throughout the motor practice blocks or across distinct apertures. As mentioned above, the visual control of wheelchair approach and passage through the aperture is an aspect as important as a priori judgments that are executed at a position that is farther away from the aperture. Thus, the variables TAU and DOT were obtained in this study in an exploratory manner to assess continuous visual judgments. In principle, the time-topassage information is continuously available from the optic flow, indicating the amount of time that remains until the aperture plane is reached. In each trial, the variable TAU is the first value of the curve (Figure 3, top right) at the moment of peak velocity (Figure 3, bottom right), expressing the instant when deceleration began. Thus, the decision on when to start to decelerate the wheelchair during the approach to the aperture was assumed to be based on TAU, the inverse of the rate of image dilation. The change in TAU over time during the deceleration phase (DOT) expresses the intensity of deceleration in terms of visual information and should be controlled to keep it constant, thus allowing safety. We expected that TAU (i.e., the time that remained until passing through the aperture) would be invariant at the onset of final deceleration across distinct velocities, apertures, and other circumstances of the task. This sort of expectation arises from studies that tested the generality of such variables as TAU and DOT. Rodrigues and colleagues (Rodrigues et al., 2006; Rodrigues et al., 2012) investigated whether TAU and DOT are invariant aspects of information used during 
a bicycle braking task. Specifically, the bicycle's initial velocity and type of trajectory were manipulated in these studies. Increased velocity and curvilinear trajectory were expected to alter the cognitive, perceptual, and motor requirements of the task to dissociate the visual control of braking from the optic flow. The results generally supported the rejection of this hypothesis, showing that these manipulations did not affect the use of optic flow (particularly the TAU and DOT variables) to control bicycle braking. The same was true for the present results. An additional point in the present study was that DOT was expected to be invariant around -.5 , according to the theory of braking (Lee, 1976). Variations of the magnitude of DOT have been debated in the literature. Bardy and Warren (1997) argued that a value of -.5 should not be interpreted as the only value that allows safe braking. Various studies have shown that the magnitude of DOT is task-dependent. Values $>-.5$ were found when the required velocity at contact was close to zero, such as in braking situations (Lee, 1976; Yilmaz \& Warren, 1995), the final phase of the task of kissing a portrait, and the phase of transport in a task of running and grasping (Wann et al., 1993). Values $<-.5$ were found when a non-zero velocity was required at contact, in contexts such as somersault landing (Lee et al., 1992) and the action of grasping (Zaal \& Bootsma, 1995). These data suggest that different DOT values may be adopted for distinct tasks depending of the type of contact desired. The mean DOT values (represented by the slope of the linear regression line in each trial) between -.547 and -.602 in the present study are compatible with this task dependency in which the final velocity that was involved in the task, after crossing the aperture, was close to zero. In summary, according to this strategy, these two invariant aspects of information would be indicative of the continuous visual judgment of apertures and its respective motor adjustments. The time-to-passage analysis included in the present study provided data that corroborated the notion that the invariance of TAU and DOT during the motor practice trials indicates that the participants used time-to-passage information during the control of wheelchair locomotion.

\section{Conclusions}

The results of the present study revealed the specificity of learning and experience in visual judgment tasks in which participants overestimated the space needed for walking and underestimated the space needed for wheelchair locomotion, independent of group. The accuracy of judgments improved from the pre-practice to post-practice condition in both groups. The motor practice to which some participants were subjected promoted a slight improvement of performance by reducing motor errors in wheelchair locomotion. During the practice trials, the participants controlled the wheelchair's final deceleration prior to reaching the aperture based on timeto-passage information. The participants also adaptively modulated wheelchair locomotion and adjusted it to the apertures that were available. The present findings of a priori visual judgments of space and continuous judgments that are necessary for wheelchair locomotion and for passing through apertures appear to support the dissociation between perception and action processes, as suggested by Milner and Goodale (1995, 2008).

\section{References}

Bardy, B. G., \& Warren, W. H., Jr. (1997). Visual control of braking in goal-directed action and sport. Journal of Sports Sciences, 15, 607-620.

Flascher, O. M., Shaw, R. E., Kader, E. E., \& Aromin, T. A. (1995) Intentional perceptual scaling means 1. In: B. G. Bardy, R. J. Bootsma, \& Y. Guiard (Eds.), Studies in perception and action III (pp. 133-136). Mahwah, NJ: Erlbaum.

Gibson, J. J. (1966). The senses considered as perceptual systems. Boston: Houghton-Mifflin Company.

Gibson, J. J. (1979). The ecological approach to visual perception. Boston: Houghton-Mifflin Company.

Higuchi, T., Takada, H., Matsuura, Y., \& Imanaka, K. (2004). Visual estimation of spatial requirements for locomotion in novice wheelchair users. Journal of Experimental Psychology: Applied, 10, 55-66.

Jiang, Y., \& Mark, L. S. (1994). The effect of gap depth on the perception of whether a gap is crossable. Perception and Psychophysics, 56, 691-700.

Kim, N. G., Turvey, M. T., \& Carello, C. (1993). Optical information about severity of upcoming contacts. Journal of Experimental Psychology: Human Perception and Performance, 19, 179-193.

Kunz, B. R., Creem-Regehr, S. H., \& Thompson, W. B. (2013). Does perceptual-motor calibration generalize across two different forms of locomotion? Investigations of walking and wheelchairs. PLoS One, 8(2), e54446.

Lee, D. N. (2009). General tau theory: evolution to date. Perception, 38, 837-858.

Lee, D. N. (1998). Guiding movement by coupling taus. Ecological Psychology, 10, 221-250.

Lee, D. N. (1976). A theory of visual control of braking based on information about time-to-collision. Perception, 5, 437-459.

Lee, D. N., Lishman, J. R., \& Thomson, J. A. (1982). Regulation of gait in long jumping. Journal of Experimental Psychology: Human Perception and Performance, 8(3), 448-459.

Lee, D. N., Young, D. S., Reddish, P. E., \& Rand, D. T. (1991). Aerial docking by hummingbirds. Naturwissenschaften, 78, 526-527.

Lee, D. N., Young, D. S., \& Rewt, D. (1992). How do somersaulters land on their feet? Journal of Experimental Psychology: Human Perception and Performance, 18, 1195-1202.

Mark, L. S. (1987). Eyeheight-scaled information about affordances: a study of sitting and stair climbing. Journal of Experimental Psychology: Human Perception and Performance, 13, 361-370.

Mark, L. S., Balliet, J. A., Craver, K. D., Douglas, S. D., \& Fox, T. (1990). What an actor must do in order to perceive the affordance for sitting. Ecological Psychology, 2, 325-366.

Maxwell, S. E., \& Delaney, H. D. (1990). Designing experiments and analyzing data: a model comparison perspective. Pacific Grove, CA: Brooks/Cole.

Milner, A. D., \& Goodale, M. A. (1995). The visual brain in action. Oxford: Oxford University Press.

Milner, A. D., \& Goodale, M. A. (2008). Two visual systems reviewed. Neuropsychologia, 46(3), 774-785.

Rodrigues, S. T., Bertoloni, G. C., Denardi, R. A., \& Ferracioli, M. C. (2006). Controle visual do início e da intensidade da freada em ciclistas: a velocidade não afeta o uso da informação de tempo para colisão. Brazilian Journal of Motor Behavior, 1(1), 64-72.

Rodrigues, S. T., Schiavon, R., \& Macegoza, J. (2012). O tipo de trajetória não afeta o controle visual da freada em ciclistas. Revista Brasileira de Educação Física e Esporte, 26(3), 473-483.

Savelsbergh, G. J. P., Dekker, L. D., Vermeer, A., \& Hopkins, B. (1998). Locomoting through apertures of different width: a study of children with cerebral palsy. Pediatric Rehabilitation, 2, 5-13.

Scott, M. A, Li, F., \& Davids, K. (1997). Expertise and regulation of the gait in the approach phase of the long jump. Journal of Sports Sciences, 15, 597-605.

Shaw, R., Flascher, O., \& Kadar, E. (1995). Dimensionless invariants for intentional systems: measuring the fit of vehicular activities to environmental layout. In: J. Flach, P. Hancock, J. Caird, \& K. 
Vicente (Eds.), Global perspectives on the ecology of humanmachine systems (pp. 293-357). Hillsdale, NJ: Erlbaum.

Stoffregen, T. A., Yang, C. M., \& Bardy, B. G. (2005). Affordance judgments and nonlocomotor body movement. Ecological Psychology, 17, 75-104.

Stoffregen, T. A., Yang, C. M., Giveans, M. R., Flanagan, M., \& Bardy, B. G. (2009). Movement in the perception of an affordance for wheelchair locomotion. Ecological Psychology, 21, 1-36.

Sun, H., \& Frost, B. J. (1998). Computation of different optical variables of looming objects in pigeon nucleus rotundus neurons. Nature Neuroscience, 1, 296-303.

Treffner, P., Barret, R., \& Petersen, A. (2002). Stability and skill in driving. Human Movement Science, 21, 749-784.

Treffner, P., Barret, R., Petersen, A., \& White, R. (2002). Active stabilisation and perceptual sensitivity in safe driving. In: Developing safer drivers and riders: Conference Proceedings (pp. 91-104). Canberra: Australian College of Road Safety.

Tresilian, J. R. (1994). Approximate information sources and perceptual variables in interceptive timing. Journal of Experimental Psychology: Human Perception and Performance, 20, 154-173.

Tresilian, J. R. (1999). Visually timed action: time-out for "tau"? Trends in Cognitive Sciences, 3, 301-310.
Wann, J. P. (1996). Anticipating arrival: is the tau-margin a specious theory? Journal of Experimental Psychology: Human Perception and Performance, 22, 1031-1048.

Wann, J. P., Edgar, P., \& Blair, D. (1993). Time-to-contact judgement in the locomotion of adults and preschool children. Journal of Experimental Psychology: Human Perception and Performance, 19, 1053-1065.

Warren, W. H., Jr., \& Whang, S. (1987). Visual guidance of walking through apertures: body-scaled information for affordances. Journal of Experimental Psychology: Human Perception and Performance, 13, 371-383.

Wichmann, F. A., \& Hill, J. N. (2001). The psychometric function: I. Fitting, sampling, and goodness of fit. Perception and Psychophysics, 63(8), 1293-1313.

Winter, D. A. (1990). Biomechanics and motor control of human movement. New York: John Wiley \& Sons.

Yilmaz, E. H., \& Warren, W. H., Jr. (1995). Visual control of braking: a test of the tau hypothesis. Journal of Experimental Psychology: Human Perception and Performance, 21, 996-1014.

Zaal, F. T. J. M., \& Bootsma, R. J. (1995). The topology of limb deceleration in prehension tasks. Journal of Motor Behavior, 27(2), 193-207. 